\title{
Health Care Resource Utilization and Costs in Patients with Chronic Myeloid Leukemia with Better Adherence to Tyrosine Kinase Inhibitors and Increased Molecular Monitoring Frequency
}

\author{
Dominick Latremouille-Viau, MSc; Annie Guerin, MSc; Patrick Gagnon-Sanschagrin, BSc; \\ Katherine Dea, MSc; Benjamin G. Cohen, MPH; and George J. Joseph, PhD, MS
}

\begin{abstract}
BACKGROUND: Frequent molecular monitoring (qPCR tests), as recommended by evidence-based monitoring guidelines, is associated with higher adherence to tyrosine kinase inhibitors (TKIs) in the management of chronic myeloid leukemia (CML); both factors have been associated with better clinical and economic outcomes.

OBJECTIVES: To (a) estimate the effect of more frequent qPCR tests on health care resource utilization (HRU) and associated costs, including direct (effect of qPCR test frequency on HRU) and indirect (through TKI adherence) effects, and (b) develop an economic model applicable to multiple clinical practice scenarios.
\end{abstract}

METHODS: Adult patients newly diagnosed with CML who started TKI firstline therapy were identified from U.S. administrative claims data (20102015). TKI adherence (medication possession ratio [MPR]), number of inpatient days, emergency room (ER) visits, outpatient service days, and mean costs per HRU event were measured during the first year of CML treatment. Direct and indirect effects of qPCR test frequency were estimated using multivariate regression models. Subsequently, an economic model was developed to assess the overall effect of varying qPCR test frequency on $\mathrm{HRU}$ and associated costs during the first year of CML treatment under different clinical practice scenarios; the scenario reported is the increase from 1 to 2 qPCR tests.

RESULTS: Of the 1,431 patients included, $36 \%$ had no qPCR tests, the average qPCR test frequency was 1.6 , and the average MPR was 0.86 during the first year of CML treatment. The direct effect of increasing qPCR test frequency by 1 was associated with $13.0 \%$ fewer inpatient days (adjusted incidence rate ratio [adjusted IRR] $=0.87 ; P=0.010$ ); $8.3 \%$ fewer ER visits (adjusted IRR $=0.92 ; P=0.043$ ); and $3.0 \%$ more outpatient service days (adjusted IRR =1.03; $P=0.002$ ). Each increase of 1 test was associated with an increase in TKI adherence by 2.2 percentage points (adjusted MPR difference $=0.022 ; P<0.001$ ). When considering the indirect effect of $\mathrm{QPCR}$ test frequency through TKI adherence, an increase of 1 qPCR test combined with an increase in TKI adherence by 2.2 percentage points was associated with a greater reduction of inpatient days from $13.0 \%$ to $15.2 \%$, ER visits from $8.3 \%$ to $8.6 \%$, and a smaller increase of outpatient service days from $3.0 \%$ to $2.6 \%$. Based on the economic model, an increase from 1 to 2 qPCR tests, considering the increase in TKI adherence, was associated with a reduction of $0.87(95 \% \mathrm{Cl}=-1.49,-0.18)$ inpatient days and $0.06(95 \%$ $\mathrm{Cl}=-0.12,0.05)$ ER visits, an increase of $0.98(95 \% \mathrm{Cl}=0.25,1.60)$ outpatient service days and a cost savings of $\$ 2,918(95 \% \mathrm{Cl}=-5,213,-349)$ per patient per year.

CONCLUSIONS: Closer alignment with the monitoring guidelines' recommended qPCR test frequency and better adherence to TKIs were associated with lower HRU and medical service costs. Managed care initiatives to increase qPCR test frequency and TKI adherence might benefit from an enhanced reduction because of the interaction between both factors.

J Manag Care Spec Pharm. 2017;23(2):214-24

Copyright $\odot 2017$, Academy of Managed Care Pharmacy. All rights reserved.

\section{What is already known about this subject}

Frequent molecular monitoring during the course of treatment of chronic myeloid leukemia (CML) is recommended by evidencebased monitoring guidelines such as the National Comprehensive Care Network guidelines.

From distinct studies, (a) frequent molecular monitoring has been associated with better adherence to treatment with tyrosine kinase inhibitors (TKIs), and (b) high adherence to TKIs and frequent molecular monitoring have been associated with better clinical and economic outcomes in the management of CML.

\section{What this study adds}

The effect of more frequent molecular monitoring was estimated, taking into consideration the indirect effect, through the improved adherence to TKIs, on health care resource utilization and associated costs to develop an economic model applicable to multiple clinical practice scenarios.

Over one third of patients with CML (36\%) received no molecular monitoring within the first year of TKI therapy.

Overall, each additional molecular monitoring test, estimated at $\$ 223$, was associated with fewer inpatient admissions, inpatient days, and emergency room visits and an increase in days with outpatient services, generating a positive economic effect of $\$ 2,918$ per patient per year.

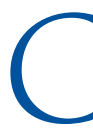
hronic myeloid leukemia (CML), a myeloproliferative disorder, represents approximately $15 \%$ of all adult leukemia cases diagnosed in the United States. ${ }^{1}$ Recent statistics on CML report that approximately 44,885 people in the United States have CML, with the incidence rate being 1.8 new cases per 100,000 individuals per year. ${ }^{2}$

In $95 \%$ of patients with CML, an abnormal chromosome, the Philadelphia chromosome, is found. It is generated by a reciprocal chromosome translocation (between 9 and 22), resulting in the fusion gene $B C R-A B L 1$ and the production of an oncogenic tyrosine kinase protein. ${ }^{3,4}$ Tyrosine kinase inhibitors (TKIs) have been the standard of care for more than a decade and are used by the vast majority of patients. ${ }^{4,5}$ TKIs, including the first-generation imatinib (introduced in 2001) and 
second-generation dasatinib and nilotinib, ${ }^{6-8}$ have improved the 8-year survival of patients newly diagnosed with CML by year of diagnosis from $42 \%-65 \%$ in $1983-2000$ to $87 \%$ since $2001 .{ }^{9}$ CML is now managed as a chronic condition.

Successful TKI therapy has the potential to reduce the disease burden of CML below the threshold of detection of hematologic and cytogenetic testing, and there is evidence that the early achievement of reduced disease burden predicts favorable long-term outcomes. ${ }^{10-12}$ Molecular monitoring, which measures $B C R-A B L 1$ transcript level using quantitative real-time polymerase chain reaction (qPCR) on the International Scale (IS), allows the detection of low levels of residual leukemia cells and provides important prognostic information for CML patients receiving TKI. ${ }^{13}$ Molecular monitoring helps determine whether a patient is responding optimally to treatment, helps identify those at risk of progression, and provides evidence regarding the need to reassess treatment or initiate second-line therapy ${ }^{14-16}$ Thus, it is important to monitor a patient's molecular response at regular intervals to gauge continuing response to TKI therapy.

Regular molecular monitoring has been incorporated into several CML treatment guidelines since 2006, including those of the European LeukemiaNet (ELN) and the U.S. National Comprehensive Care Network (NCCN). ${ }^{16,17}$ The NCCN guidelines recommend molecular monitoring every 3 months after TKI initiation (except at the third and sixth months, where cytogenetic monitoring may be used if molecular monitoring using the IS is not available), every 3 months for 2 years, and every 3-6 months in the following years after complete cytogenetic response (CCyR) achievement. ${ }^{17}$ If $B C R-A B L 1$ transcript levels increase by 1 log after achieving a major molecular response, the NCCN guidelines recommend testing to be repeated after 1-3 months.

Despite these recommendations, several studies have demonstrated that in clinical practice, patients with CML are often monitored less frequently for molecular response than is recommended. ${ }^{12,18-20}$ Payers could have the capacity to encourage the frequency of molecular monitoring as routine oncology practice by providing supportive measures to help physicians comply with evidence-based guidelines. However, in the U.S., few payers proactively encourage the management of molecular response monitoring in patients with CML, although more frequent monitoring has been associated with improved adherence to TKIs, ${ }^{18}$ lower risk of progression, ${ }^{12}$ and lower health resource utilization (HRU) and costs. ${ }^{19}$ In addition, higher adherence to TKIs, which may result from more frequent molecular monitoring, has also been shown to reduce HRU.18,21

Because of the importance of preventing CML disease progression and providing patients with the optimal benefits from treatment, there is a need to better understand the effect of the frequency of molecular monitoring on clinical outcomes in real-world practice. Thus, the first objective of this study was to estimate the overall effect of more frequent molecular monitoring on HRU in adult patients newly diagnosed with CML, including direct (the effect of qPCR test frequency on HRU) and indirect (through improved TKI adherence) effects. The second objective was to develop an economic model to illustrate the overall effect of more frequent molecular monitoring on HRU and associated costs, including the indirect effect through TKI adherence, applicable for multiple scenarios in clinical practice, assuming that the increased frequency of molecular monitoring would be associated with lower HRU, which in turn may translate into lower costs.

\section{Methods}

\section{Data Source}

Two large U.S. administrative claims databases spanning January 1, 2006-June 30, 2015, were used to identify commercially insured adult patients newly diagnosed with CML who were initiated on first-line TKI therapy. These databases include patient-level data from over 200 health plans and annually represent over 115 million enrollees from all U.S. census regions. Data were de-identified to comply with the confidentiality requirements of the Health Insurance Portability and Accountability Act (HIPAA); therefore, no institutional review was required.

\section{Study Design and Sample Selection}

The design was a retrospective cohort study. The index date was the first TKI (i.e., imatinib, dasatinib, or nilotinib) prescription fill date, and the baseline period was the 6 months before the index date. The study period was the 12 months following the index date and was used to measure outcomes. The observation period was the 13 months following the index date and was used to measure frequency of molecular monitoring. The thirteenth month following the index date was used to allow for the observation of a potential fourth molecular monitoring test that may have been delayed by a few days, since molecular monitoring is recommended every 3 months when patients respond to TKI therapy. ${ }^{17}$

From the 2 claims databases, patients were included if they (a) were aged $\geq 18$ years at the index date; (b) received imatinib, dasatinib, or nilotinib on or after October 28, 2010, the date when the 3 TKIs were available as first-line therapy for CML (i.e., after U.S. Food and Drug Administration approval of dasatinib [October 28, 2010], imatinib [May 10, 2001], and nilotinib [June 17, 2010]); ${ }^{6-8}$ (c) had the first diagnosis for CML observed during the baseline period or the month following the index date; and (d) had continuous health plan coverage during the baseline and observation periods.

Patients were excluded if they had (a) received omacetaxine mepesuccinate, bosutinib, or ponatinib before or on the index date; (b) a procedure for hematopoietic stem cell transplantation during the baseline period or at the index date; (c) used 
chemotherapy (except hydroxyurea) during the baseline period or at the index date; (d) a CML remission/relapse diagnosis before or on the index date; (e) an indicator of clinical trial enrollment during the baseline or observation periods; (f) $>4$ molecular monitoring tests during the observation period; or (g) a diagnosis for metastatic cancer or solid tumor during the baseline period or at the index date (Appendix A, available in online article). Reasons for having molecular monitoring were not available in the database, and considering the recommendation by NCCN guidelines for molecular monitoring, it was assumed that patients receiving $>4$ tests in the first 13 months of TKI initiation were likely to present unusual clinical profiles and were excluded. ${ }^{12,17-19}$

\section{Measures and Outcomes}

The frequency of molecular monitoring was measured during the observation period and was identified using procedure codes recorded in medical claims for qPCR testing (Appendix B, available in online article). Because specific Current Procedural Terminology procedure codes to identify qPCR tests were effective starting January $1,2013,{ }^{22}$ these specific codes were used after January 1, 2013, and groups of related procedure codes, $\geq 30$ days apart, were used to identify molecular monitoring before 2013.

The medication possession ratio (MPR) was used to assess adherence to any TKI therapy (i.e., imatinib, dasatinib, nilotinib, bosutinib, or ponatinib) during the study period. The MPR was the number of days of TKI supplied during the study period divided by the number of calendar days in the study period (i.e., 365 days), and was truncated at 1 if the total number of days of TKI supply exceeded 365 days (i.e., overlapping prescription fills). ${ }^{23}$ Adherence to TKIs was analyzed as a continuous variable of MPR, theoretically ranging from 0 to 1 .

The following HRU outcomes were measured during the study period and reported per patient per year: number of inpatient (IP) admissions and IP days; emergency room (ER) visits; and days with outpatient (OP) services (e.g., office visits, any laboratory services including molecular monitoring tests, and home care services). Associated health care costs were measured during the study period; mean costs were calculated by HRU event (i.e., 1 IP day, 1 ER visit, 1 day with OP services) considering each event that occurred during the study period. Medical service costs were measured from a U.S. payer perspective (i.e., amount reimbursed by the commercial plan and coordination of benefits) and adjusted to 2015 U.S. dollars using the Consumer Price Index, medical care component. ${ }^{24}$

\section{Statistical Methods}

A zero-inflated negative binomial (ZINB) regression model was used to estimate the effect of more frequent molecular monitoring and higher adherence to TKIs on HRU (i.e., the direct effect of molecular frequency and adherence to TKIs on HRU in the economic model below). The first part of the ZINB model was a general linear model (GLM) with a log link and a logit distribution, and the second part was a GLM with a log link and a negative binomial distribution (due to the overdispersion confirmed by a chi-square test ${ }^{25}$ ); incidence rate ratios (IRRs) were reported. Goodness of fit was assessed using the Vuong test for each HRU outcome. ${ }^{26} \mathrm{P}$ values with $95 \%$ confidence intervals (CIs) were estimated using a robust standard error. ${ }^{27}$

The effect of molecular monitoring frequency on adherence to TKIs (i.e., the indirect effect through adherence to TKIs in the economic model below) was estimated using ordinary least-squares regression models (OLS), and the difference in MPR was reported. OLS assumptions for unbiased and robust estimates were met. ${ }^{28} \mathrm{P}$ values and 95\% CIs were estimated using a robust standard error. ${ }^{27}$

All regression models were adjusted for the following a priori selected potential confounding factors: age, sex, year of the index date, U.S. census region, health plan type, whether patients started TKI therapy on the recommended dose for CML-chronic phase ${ }^{6-8}$ Darkow CML Complexity Index score (which uses reported diagnoses of associated complications, comorbidities, or adverse events to categorize the difficulty of managing the patients' disease as mild, moderate, or severe), ${ }^{29}$ and Charlson-Quan Comorbidity Index (CCI) score. ${ }^{30}$

\section{Economic Model}

Based on the results of the statistical analyses, an economic model was developed to estimate the overall effect of the frequency of molecular monitoring on HRU and associated costs, considering the indirect effect through adherence to TKIs on HRU, over the first year of CML management.

Model inputs included frequency of molecular monitoring, adherence to TKIs, and mean cost per HRU event. The model output allowed an estimation of the putative overall effect of more frequent molecular monitoring for multiple scenarios in clinical practice. Using estimated parameters of the ZINB models, the number of HRU events was estimated at a predetermined frequency of molecular monitoring (e.g., 1 test), holding the TKI adherence level and other variables at their mean values. This was compared with the number of HRU events estimated at an increased test frequency (e.g., 2 tests), considering the increase in TKI adherence level (using the estimated effect on MPR of the OLS model) and holding all other variables at their mean values. The difference in the number of HRU events associated with an increase in the number of molecular monitoring tests was translated into medical service costs by multiplying the difference in the number of HRU events by the mean cost of the corresponding HRU outcome. The 95\% CIs of the differences in the number of HRU events and costs, considering the direct and indirect effects, were estimated using a nonparametric bootstrap resampling technique with 499 iterations. ${ }^{31,32}$ 
To illustrate the overall effect of more frequent molecular monitoring on each HRU outcome, stratified by direct and indirect effects, 2 potential scenarios in clinical practice settings are presented: (1) an increase from 1 to 2 molecular monitoring tests, and (2) an increase from 2 to 4 molecular monitoring tests over the first year of CML treatment.

Statistical significance was set at the 0.05 level. All data analyses were performed using SAS, version 9.3 (SAS Institute, Cary, NC).

\section{Results}

A total of 1,431 adult patients newly diagnosed with CML met the sample selection criteria (Figure 1), composed of 668 (47\%) patients who started first-line TKI therapy with imatinib, 443 (31\%) who started with dasatinib, and 320 (22\%) who started with nilotinib (Table 1). Patient mean age was 54 years, with $19 \%$ of patients aged $\geq 65$ years, and $47 \%$ of patients were female. During the study period, most (42\%) patients had mild Darkow complexity (36\% moderate and $22 \%$ severe), and the average CCI was 2.1. The 5 most prevalent comorbidities were hypertension (46\%), hyperlipidemia (39\%), anemia (34\%), diabetes (24\%), and cardiovascular disease (19\%).

During the first year of CML management, $36 \%$ of patients had no molecular monitoring tests, $16 \%$ had $1,15 \%$ had $2,18 \%$ had 3 , and $16 \%$ had 4 (Table 1); on average, patients had 1.6 molecular monitoring tests. The proportion of patients without any molecular monitoring tests tended to decrease with later index date years (i.e., 46\% in 2010-2011, 27\% in 2013-2014). The average adherence to TKIs was 0.86 , and $75 \%$ of patients had an adherence $>0.80,{ }^{18,23,33,34}$ meaning that the majority of patients were considered as adherent to TKI therapy and used it continuously through the first year of CML treatment. The mean costs were estimated to be $\$ 3,660$ per IP day, $\$ 848$ per ER visit, and $\$ 313$ per day with OP services.

When assessing the direct effect of molecular monitoring frequency on HRU outcomes, an increase of 1 molecular monitoring test was associated with a reduction in the number of IP admissions by $11.6 \%$ (adjusted IRR $=0.8839 ; P=0.012$ ), IP days by $13.0 \%$ (adjusted IRR $=0.8701 ; P=0.010$ ), ER visits by $8.3 \%$ (adjusted IRR $=0.9167 ; P=0.043$ ), and an increase in the number of days with OP services by 3.0\% (adjusted IRR $=1.0296$; $P=0.002$; Figure 2). Concurrently, from the same regression model, when assessing the direct effect of adherence to TKIs on HRU outcomes, an increase in adherence to TKIs by 1 unit (i.e., 1 percentage point-the difference in MPR level) was associated with a reduction in the number of IP admissions by $1.3 \%$ (adjusted IRR $=0.9865 ; P<0.001$ ), the number of IP days by $1.1 \%$ (adjusted IRR $=0.9885 ; P<0.001$ ), and the number of ER visits and days with OP services by a nonstatistically significant $0.1 \%$ (adjusted IRR $=0.9988, P=0.640$ and adjusted IRR $=0.9985, P=0.060$, respectively; results not presented).
When assessing the effect of molecular monitoring frequency on adherence to TKIs, each increase of 1 molecular monitoring test was associated with an increase in adherence to TKIs by 2.2 units (i.e., 2.2 percentage points; adjusted difference in MPR level of 0.0221; $P<0.001$; Figure 3). Consequently, when also considering the indirect effect of molecular monitoring frequency through improved adherence to TKIs on HRU outcomes, an increase of 1 molecular monitoring test combined with an increase of adherence to TKIs by 2.2 percentage points was associated with a greater reduction of the number of IP admissions from $11.6 \%$ to $14.2 \%$, IP days from $13.0 \%$ to $15.2 \%$, a greater reduction of ER visits from $8.3 \%$ to $8.6 \%$, and a smaller increase in the number of days with OP services from $3.0 \%$ to $2.6 \%$. The indirect effect of molecular monitoring test frequency through adherence to TKIs accounted for $18.4 \%$, $14.5 \%$, and $2.8 \%$ of the reduction in IP admissions, IP days, and ER visits, respectively.

Based on the economic model, increasing test frequency from 1 to 2 tests for a patient over the first year of CML management is expected to result in the following changes in HRU outcomes and costs per newly diagnosed CML patient per year: (a) a reduction of 0.04 IP admissions or 0.87 IP days, resulting in a cost savings of $\$ 3,175$; (b) a numerical, but nonstatistically significant, reduction of 0.06 ER visits, resulting in a cost savings of $\$ 50$; (c) an increase of 0.98 days with OP services, resulting in a cost increase of $\$ 307$; and (d) a total cost savings of $\$ 2,918$ (Table 2). A second scenario, modeling an increase from 2 to 4 tests for a patient over the first year of CML management is expected to result in the following changes per newly diagnosed CML patient per year: (a) a reduction of 0.07 IP admissions or 1.4 IP days, resulting in a cost savings of $\$ 5,106$; (b) a numerical, but nonstatistically significant, reduction of 0.1 ER visits, resulting in a cost savings of $\$ 87$; (c) an increase of 2.05 days with OP services, resulting in a cost increase of $\$ 640$; and (d) a total cost savings of $\$ 4,554$ (Table 2). For a health plan of 1 million beneficiaries, and based on a CML annual incidence of 1.8 per 100,000 individuals, ${ }^{2}$ the overall effect of increasing the molecular monitoring frequency from 1 to 2 and from 2 to 4 would be expected to lead to an estimated cost savings of $\$ 52,530$ and $\$ 81,971$, respectively, over a l-year period.

\section{Discussion}

The benefits of an adequate frequency of molecular monitoring in CML patients have been demonstrated in a number of studies. ${ }^{10-12,18,19}$ However, significant barriers to adherence to evidence-based CML monitoring guidelines exist. ${ }^{12,35,36}$ In this study, we found that $36 \%$ of patients had no molecular monitoring test within the first year of CML management, and although this proportion decreased with more recent index dates, there remained a substantial proportion of patients who received no molecular tests in the first year. According to a 
Health Care Resource Utilization and Costs in Patients with Chronic Myeloid Leukemia with

Better Adherence to Tyrosine Kinase Inhibitors and Increased Molecular Monitoring Frequency

\section{FIGURE 1 Sample Selection of Patients Newly Diagnosed with CML Receiving First-Line TKI Therapy}

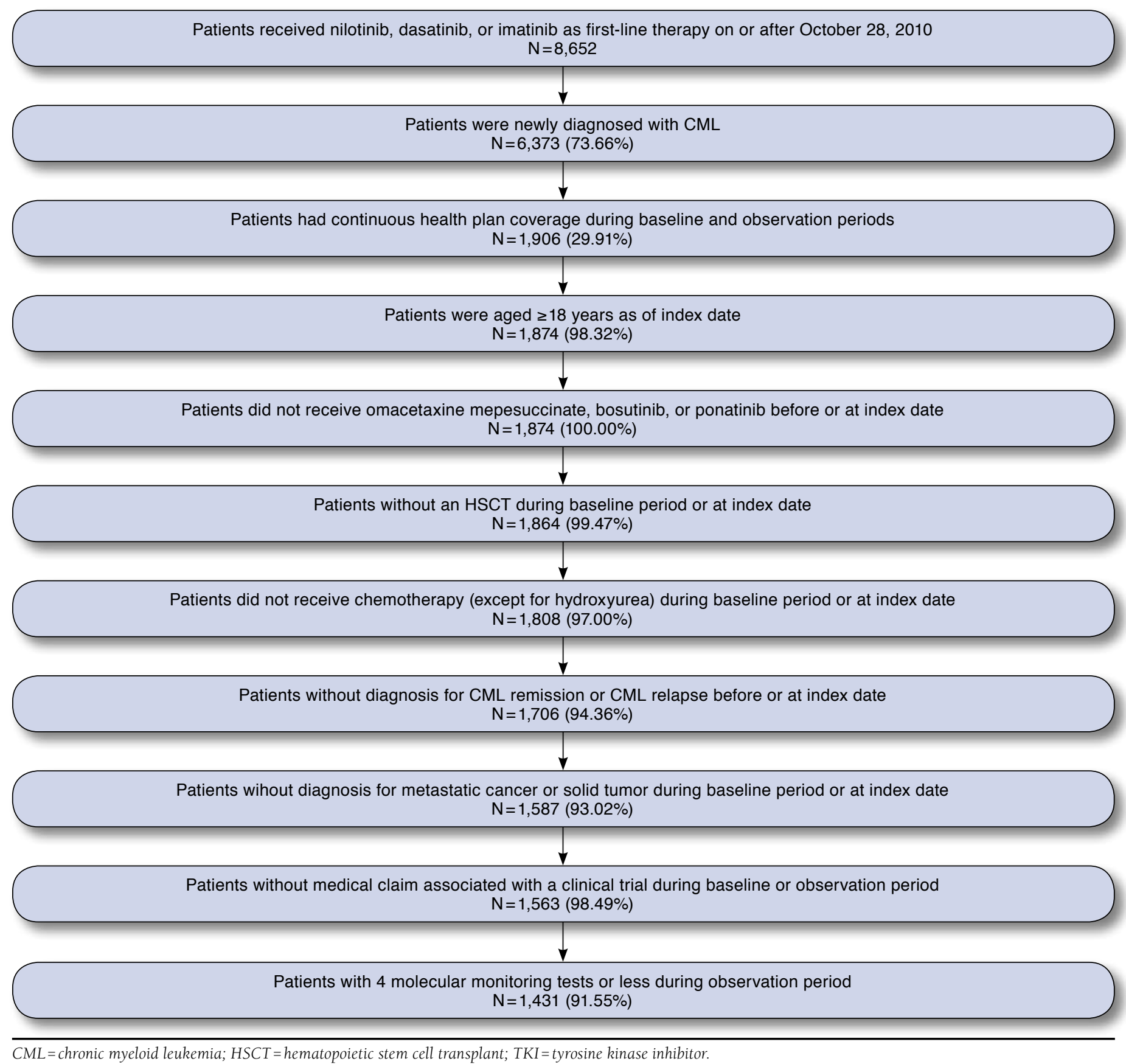

recent study by Goldberg et al. (2015), the main obstacles for physicians to adhere to monitoring guidelines include resource and accessibility barriers regarding molecular response testing, unfamiliarity with molecular testing, and a lack of agreement with monitoring recommendations. ${ }^{37}$ The findings of this study add to the existing body of evidence by demonstrating that more frequent molecular monitoring among patients with CML, in closer alignment with NCCN guidelines, is associated with lower HRU and associated costs. These findings also support the need for consideration of U.S. payer programs to improve adherence to evidence-based CML monitoring guidelines, which in turn would improve quality of CML patient 


\section{TABLE 1 Characteristics of Patients Newly Diagnosed with CML Receiving First-Line TKI Therapy}

Total number of patients

At the Index Date

Demographic characteristics

Age at the index date, years, mean \pm SD [median]

$$
65+\text { years, } \mathrm{n}(\%)
$$

Female, n (\%)

Health plan type, ${ }^{\mathrm{a}} \mathrm{n}(\%)$

Low flexibility plan

Middle flexibility plan

High flexibility plan

Region of residence, $\mathrm{n}(\%)$

\begin{tabular}{|c|c|c|}
\hline South & 530 & $(37.0)$ \\
\hline Northeast & 281 & $(19.6)$ \\
\hline Midwest & 204 & $(14.3)$ \\
\hline West & 191 & (13.3) \\
\hline North Central & 198 & $(13.8)$ \\
\hline Unknown & 27 & $(1.9)$ \\
\hline \multicolumn{3}{|l|}{ Index year, n (\%) } \\
\hline 2010 & 91 & $(6.4)$ \\
\hline 2011 & 483 & $(33.8)$ \\
\hline 2012 & 440 & $(30.7)$ \\
\hline 2013 & 359 & $(25.1)$ \\
\hline 2014 & 58 & $(4.1)$ \\
\hline \multicolumn{3}{|l|}{ Index treatment, $\mathbf{n}(\%)$} \\
\hline Imatinib & 668 & $(46.7)$ \\
\hline $\begin{array}{l}\text { Started on lower starting dose } \\
(200 \mathrm{mg} / \text { day- } 300 \mathrm{mg} / \text { day })\end{array}$ & 33 & $(4.9)$ \\
\hline $\begin{array}{l}\text { Started on recommended starting dose } \\
(400 \mathrm{mg} / \text { day })\end{array}$ & 605 & $(42.3)$ \\
\hline $\begin{array}{l}\text { Started on higher starting dose } \\
(500 \mathrm{mg} / \text { day- } 1,000 \mathrm{mg} / \text { day })\end{array}$ & 30 & $(4.5)$ \\
\hline Dasatinib & 443 & $(31.0)$ \\
\hline $\begin{array}{l}\text { Started on lower starting dose } \\
(20 \mathrm{mg} / \text { day- } 90 \mathrm{mg} / \text { day })\end{array}$ & 14 & $(3.2)$ \\
\hline $\begin{array}{l}\text { Started on recommended starting dose } \\
\text { (100 mg/day) }\end{array}$ & 404 & $(28.2)$ \\
\hline $\begin{array}{l}\text { Started on higher starting dose } \\
\text { (from } 140 \mathrm{mg} / \text { day- } 210 \mathrm{mg} / \text { day) }\end{array}$ & 25 & $(5.6)$ \\
\hline Nilotinib & 320 & $(22.4)$ \\
\hline $\begin{array}{l}\text { Started on lower starting dose } \\
(150 \mathrm{mg} / \text { day- } 400 \mathrm{mg} / \text { day })\end{array}$ & 20 & $(6.3)$ \\
\hline $\begin{array}{l}\text { Started on recommended starting dose } \\
(600 \mathrm{mg} \text { /day) }\end{array}$ & 247 & $(17.3)$ \\
\hline $\begin{array}{l}\text { Started on higher starting dose } \\
(650 \mathrm{mg} / \text { day- } 1,000 \mathrm{mg} / \text { day })\end{array}$ & 53 & $(16.6)$ \\
\hline
\end{tabular}

During Study Period

Darkow Disease Complexity Index, ${ }^{29} \mathrm{n}(\%)$

\begin{tabular}{l|c}
\hline Mild & $601 \quad(42.0)$ \\
\hline Moderate & $509(35.6)$ \\
\hline Severe & $321 \quad(22.4)$ \\
\hline $\begin{array}{l}\text { Number of qPCR monitoring tests, } \\
\text { mean } \pm \text { SD [median] }\end{array}$ & $1.61 \pm 1.50[1]$ \\
\hline 0 qPCR, n (\%) & $517 \quad(36.1)$ \\
\hline 1 qPCR, n (\%) & $226 \quad(15.8)$ \\
\hline 2 qPCR, n (\%) & $208 \quad(14.5)$ \\
\hline 3 qPCR, n (\%) & $257 \quad(18.0)$ \\
\hline 4 qPCR, n (\%) & $223 \quad(15.6)$ \\
\hline $\begin{array}{l}\text { Adherence to TKI therapy, MPR, } \\
\text { mean } \pm \text { SD [median] }\end{array}$ & $0.86 \pm 0.22$ \\
\hline$\geq 0.80, n$ (\%) & $1,069 \quad(74.7)$ \\
\hline CCI ${ }^{30}$ (excluding CML), mean \pm SD [median] & $2.10 \pm 1.95[2]$ \\
\hline 10 most prevalent comorbidities, $\mathbf{n}(\%)$ & \multicolumn{2}{|c}{6}
\end{tabular}

10 most prevalent comorbidities, $\mathrm{n}(\%)$

\begin{tabular}{|c|c|}
\hline Hypertension & $654 \quad(45.7)$ \\
\hline Hyperlipidemia & $(39.3)$ \\
\hline Anemia & $(34.3)$ \\
\hline Diabetes & $(24.2)$ \\
\hline Cardiovascular disease & $(19.4)$ \\
\hline Chronic pulmonary disease & $(15.6)$ \\
\hline Fluid electrolyte disorder & $214 \quad(15.0)$ \\
\hline Coagulopathy & $198 \quad(13.8)$ \\
\hline Hypothyroidism & $188 \quad(13.1)$ \\
\hline Valvular disease & $(11.0)$ \\
\hline \multicolumn{2}{|c|}{ Annual health care resource utilization, mean \pm SD [median] } \\
\hline IP admissions & $0.36 \pm 0.97[0]$ \\
\hline IP days & $3.10 \pm 12.79[0]$ \\
\hline ER visits & $0.71 \pm 2.09[0]$ \\
\hline Days with OP services & $28.60 \pm 19.95[25]$ \\
\hline \multicolumn{2}{|c|}{ Health care cost per event, mean \pm SD [median] } \\
\hline IP costs & $\begin{array}{c}\$ 32,496 \pm 42,736 \\
{[16,335]}\end{array}$ \\
\hline IP day costs & $\begin{array}{c}\$ 3,660 \pm 4,672 \\
{[2,262]}\end{array}$ \\
\hline ER costs & $\begin{array}{c}\$ 848 \pm 1,481 \\
{[504]}\end{array}$ \\
\hline OP costs & $\begin{array}{c}\$ 313 \pm 443 \\
{[197]}\end{array}$ \\
\hline
\end{tabular}

aPlan flexibility is based on whether the primary care giver is assigned and whether there is patient incentive to use a certain provider.

$C C I=$ Charlson-Quan Comorbidity Index; $C M L=$ chronic myeloid leukemia; $E R=$ emergency room; $I P=$ inpatient; $M P R=$ medication possession ratio; $O P=o u t p a t i e n t ;$ $q P C R=$ quantitative real-time polymerase chain reaction; $S D=$ standard deviation; TKI = tyrosine kinase inhibitor.

care and clinical outcomes. Since payers may be able to develop initiatives to increase the frequency of molecular response monitoring in routine oncology practice, the economic model built in this study describes the magnitude of the possible positive economic effect.
The findings of this study are consistent with several previous studies. A first chart review study, based on data from 2006-2010, similarly reported that only $25 \%$ of patients with CML had a molecular monitoring test by 3 months, 45\% between 4-6 months, and 65\% between 7-12 months. ${ }^{20}$ By 12 


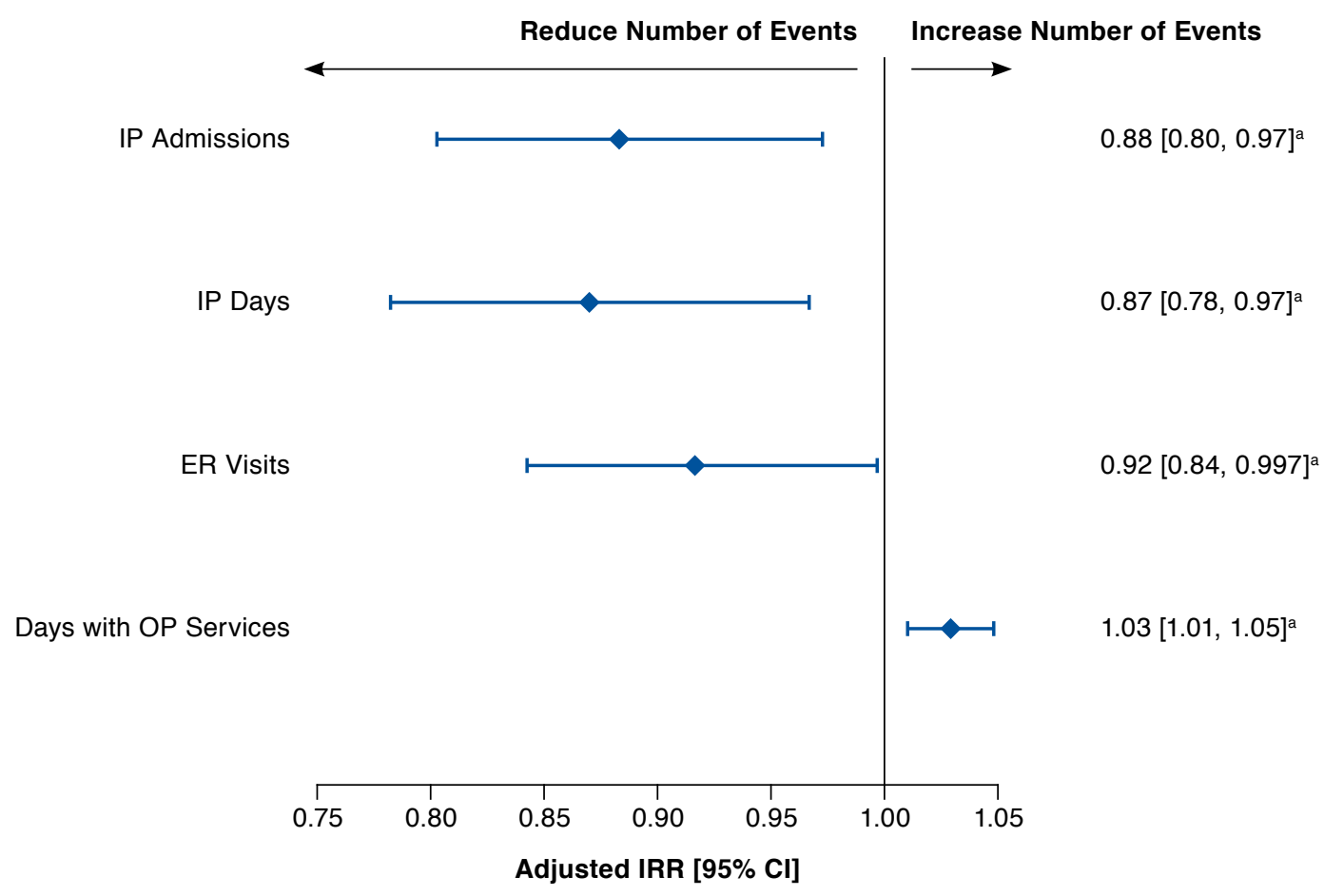

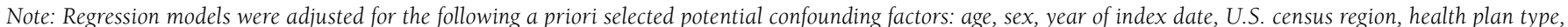

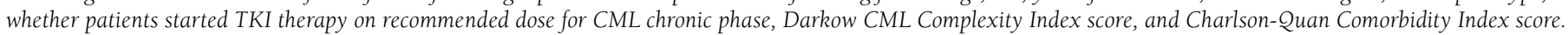
aSignificant at 5\% level $(P<0.05)$. IP admission, $P=0.012$; IP days, $P=0.010 ; E R$ visits, $P=0.043$; days with $O P$ services, $P=0.002$.

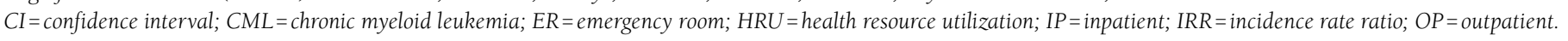

months, $72 \%$ of patients had at least 1 molecular monitoring test; $28 \%$ had never received testing in the first year. Another chart review study, using data from 2006-2011, reported that while patients with CML were in chronic phase and on TKI therapy, $13 \%$ of patients had no molecular monitoring tests; $41 \%$ had an average of $1-2$ tests per year; and $46 \%$ had an average of 3-4 tests per year. ${ }^{12}$ Finally, a claims database analysis of patients with CML who initiated a TKI therapy between 20072011 showed that over the first year following the TKI initiation, $41 \%$ of patients with CML had no molecular monitoring tests; $32 \%$ had $1-2$ tests, and $27 \%$ had $3-4$ tests. ${ }^{19}$

In the current study, more frequent molecular monitoring was associated with a reduction in HRU events, consistent with the findings of a previous retrospective claims analysis reporting a 44\% reduction in IP admissions, and lower costs, among CML patients with 3-4 tests compared with patients with no tests in the first year. ${ }^{19}$ In addition, we found that higher adherence to TKI therapy was associated with fewer IP visits and days, similar to a previous retrospective claims study among a population of privately insured patients with CML. ${ }^{21}$ The current study contributes to the literature by providing an overview of the direct and indirect effects of more frequent molecular monitoring on economic outcomes. When combining the direct and indirect effects, the total effect on HRU and health care cost is enhanced. For example, our study sample had an average of 1.6 molecular monitoring tests over the first year of CML management. Based on our economic model, the effect of increasing the frequency of testing from 2 to 4 would result in a cost savings of $\$ 4,554$ per patient, primarily driven by fewer IP days. Thus, a better alignment of the frequency of molecular monitoring with the NCCN guideline-recommended levels would result in greater direct and indirect effects, leading to substantial cost savings. Each additional molecular monitoring test, estimated at $\$ 223$ (i.e., representing $\$ 307$ in days with OP services in this study), ${ }^{38}$ would have the potential to generate a positive economic effect of $\$ 2,918$ per patient per year.

The observed reduction in HRU resulting from increased testing frequency may have several explanations. Molecular response alerts health care providers of CML disease progression, so more frequent monitoring presents more opportunities 


\section{FIGURE 3 Effect of More Frequent Molecular Monitoring on Level of Adherence to TKI Therapy over} First Year of CML Management in Newly Diagnosed CML Patients

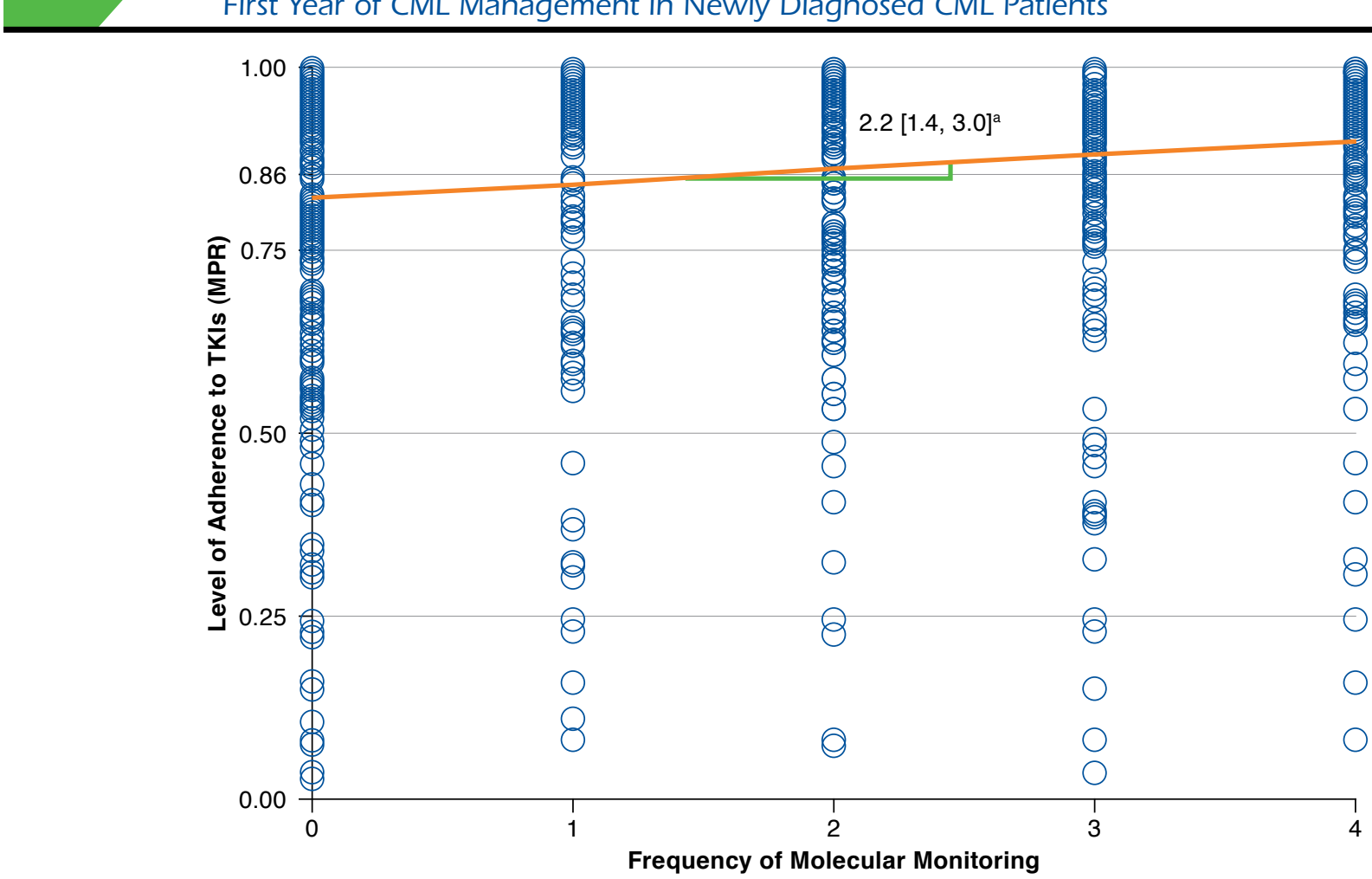

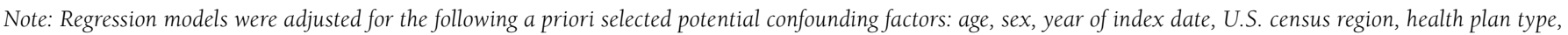

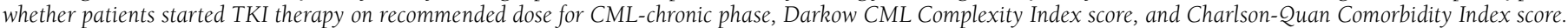
aThe estimated difference in the level of adherence to TKI therapy was 2.2 percentage points (i.e., adjusted difference in MPR of 0.022 ; P<0.001). The robustness of the estimated difference in MPR was tested using a fractional logistic regression model with robust standard error, and the results remained constant. $C M L=$ chronic myeloid leukemia; $M P R=$ medication possession ratio; TKI=tyrosine kinase inhibitor.

to determine if a patient is responding optimally and whether there is a need to reassess therapy. ${ }^{10}$ The high sensitivity of molecular monitoring, combined with the recommended testing frequency per NCCN guidelines, allows providers to monitor the achievement and maintenance of optimal treatment response, note increases in BRC-ABL1 transcript levels (a sign of potential resistance/treatment failure), and identify treatment nonadherence. ${ }^{39,40}$ Response to TKI therapy is the most important prognostic factor for disease progression and can be predicted as early as 3 months from therapy initiation. ${ }^{41,42}$ Early detection of progression or nonadherence may allow the provider to intervene quickly to minimize the potential effect on long-term and progression-free survival. ${ }^{10,11,17,41-43}$

The high proportion of patients who fail to receive any molecular monitoring tests within the first year of CML treatment signals that some providers may not appreciate the need for regular, consistent molecular monitoring or may not fully appreciate the importance of monitoring after CCyR achievement. ${ }^{36}$ There may also be a lack of familiarity regarding how frequently monitoring should be performed or how to interpret and act on test results..$^{13,37,44,45}$ For these reasons, measures that support more frequent molecular monitoring are needed to help achieve the recommended testing frequency per NCCN guidelines and encourage a proactive strategy for CML management. ${ }^{17,36}$ In addition to multifaceted educational programs aimed at raising provider awareness of routine monitoring (such as point-of-care computerized reminders), other initiatives from payers to encourage regular monitoring are warranted to improve quality of CML care. The economic model built in this study has the flexibility to be adaptive to the specific characteristics of the CMS population within individual health plans and could be used to illustrate the effect of more frequent molecular monitoring on HRU and associated health care costs of each health plan. 
TABLE 2 Potential Effect of More Frequent Molecular Monitoring on HRU Outcomes and Health Care Costs Per Newly Diagnosed CML Patients over First Year of CML Management

\begin{tabular}{|c|c|c|c|c|c|}
\hline & \multicolumn{5}{|c|}{ Adjusted Difference in Number of HRU Events/Costs } \\
\hline & \multirow{2}{*}{$\begin{array}{c}\text { Direct Effect }{ }^{\mathrm{a}} \\
\text { HRU Event }\end{array}$} & \multirow{2}{*}{$\begin{array}{c}\text { Indirect Effect } \\
\text { HRU Event }\end{array}$} & \multicolumn{3}{|c|}{ Overall Effect ${ }^{c}$} \\
\hline & & & HRU Event $[95 \% \mathrm{CI}]$ & Cost, USI & $2015[95 \% \mathrm{CI}]$ \\
\hline \multicolumn{6}{|c|}{ A. Increasing molecular monitoring frequency from 1 to 2} \\
\hline IP admissions & -0.034 & -0.008 & $-0.04 \quad[-0.08,-0.010]$ & \multirow{2}{*}{$-\$ 3,175$} & \multirow{2}{*}[-5,438,-677]{} \\
\hline IP days & -0.74 & -0.13 & $-0.87 \quad[-1.49,-0.18]$ & & \\
\hline ER visits & -0.06 & $<-0.01$ & $-0.06 \quad[-0.12,0.05]$ & $-\$ 50$ & {$[-99,41]$} \\
\hline Days with OP services & 1.11 & -0.13 & {$[0.25,1.60]$} & $\$ 307$ & {$[80,501]$} \\
\hline Total cost & & & & $-\$ 2,918$ & {$[-5,213,-349]$} \\
\hline \multicolumn{6}{|c|}{ B. Increasing molecular monitoring frequency from 2 to 4} \\
\hline IP admissions & -0.057 & -0.013 & $-0.07 \quad[-0.12,-0.02]$ & \multirow{2}{*}{$-\$ 5,106$} & \multirow{2}{*}[-7,670,-1,312]{} \\
\hline IP days & -1.21 & -0.19 & $-1.40 \quad[-2.10,-0.36]$ & & \\
\hline ER visits & -0.10 & $<-0.01$ & $-0.10 \quad[-0.18,0.11]$ & $-\$ 87$ & {$[-156,92]$} \\
\hline Days with OP services & 2.31 & -0.26 & {$[0.52,3.43]$} & $\$ 640$ & {$[162,1,072]$} \\
\hline Total cost & & & & $-\$ 4,554$ & {$[-7,203,-587]$} \\
\hline \multicolumn{6}{|c|}{$\begin{array}{l}\text { Note: Regression models were adjusted for the following a priori selected potential confounding factors: age, sex, year of index date, U.S. census region, health plan type, } \\
\text { whether patients started TKI therapy on recommended dose for CML-chronic phase, Darkow CML Complexity Index score, and Charlson-Quan Comorbidity Index score. } \\
\text { a Direct effect of qPCR test frequency on HRU alone. } \\
\text { bIndirect effect of qPCR test frequency through TKI adherence; increasing qPCR test frequency increased TKI adherence level, which in turn reduced HRU events. } \\
\text { cSum of direct and indirect effects. } \\
C I=\text { confidence interval; CML=chronic myeloid leukemia; ER=emergency room; HRU =health resource utilization; IP=inpatient; OP=outpatient; qPCR=quantitative } \\
\text { real-time polymerase chain reaction; TKI = tyrosine kinase inhibitor; USD =U.S. dollars. }\end{array}$} \\
\hline
\end{tabular}

\section{Limitations}

The results of this study should be interpreted in the context of its limitations, which are inherent to the data sources. First, the studied sample was limited to commercially insured employees and their dependents diagnosed with CML, so generalizability to the overall CML population may be limited. Second, measures of disease severity such as the Sokal score or performance status, ${ }^{46,47}$ phase of CML (chronic phase, accelerated phase, or blast $\mathrm{crisis}^{17}$ ), and laboratory test results (including the availability of molecular monitoring using the IS) were not available in the data. Third, there was no procedure code to identify molecular monitoring (i.e., qPCR tests) in claims data before January 1,2013 . Therefore, an algorithm was developed to identify these tests. Fourth, 2 databases were stacked to increase the sample size. According to data use agreements, data cannot be combined in any manner that would allow for re-identification of payers or plans, and per HIPAA requirements, it is forbidden to assess the potential overlap of patients between the 2 databases; however, the potential overlap, if any, was insignificant. Fifth, this study demonstrated the association between molecular monitoring frequency and adherence to TKI therapy and economic outcomes, not causation.

\section{Conclusions}

Closer alignment with the NCCN guideline-recommended molecular monitoring frequency and better adherence to TKIs were associated with a reduction in HRU events and costs among newly diagnosed adult patients with CML receiving first-line TKI therapy. Managed care initiatives or incentives to increase molecular monitoring frequency and improve adherence to TKIs have the potential to generate a positive economic effect.

\section{Authors}

DOMINICK LATREMOUILLE-VIAU, MSC; ANNIE GUERIN, MSc; PATRICK GAGNON-SANSCHAGRIN, BSc; and KATHERINE DEA, MSc, Analysis Group, Montreal, Canada. BENJAMIN G. COHEN, MPH, and GEORGE J. JOSEPH, PhD, MS, Novartis Pharmaceuticals, East Hanover, New Jersey.

AUTHOR CORRESPONDENCE: Dominick Latremouille-Viau, Senior Economist, Analysis Group, 1000 De La Gauchetière West, Ste. 1200, Montreal, QC H3B4W5, Canada. Tel.: +1.514.394.4431; E-mail: Dominick.Latremouille-Viau@analysisgroup.com.

\section{DISCLOSURES}

This study was funded by Novartis Pharmaceuticals, which was involved in all stages of the study and in the decision to submit the report for publication. Latremouille-Viau, Guerin, Gagnon-Sanschagrin, and Dea are employees of Analysis Group, which received consulting fees from Novartis Pharmaceuticals for work on this study. Joseph is an employee of Novartis Pharmaceuticals and owns stock in Amgen and Pfizer. Cohen was an employee of Novartis Pharmaceuticals at the time of this study. 


\section{Health Care Resource Utilization and Costs in Patients with Chronic Myeloid Leukemia with Better Adherence to Tyrosine Kinase Inhibitors and Increased Molecular Monitoring Frequency}

Portions of this study were presented online (beginning May 20,2016) as part of the American Society of Clinical Oncology (ASCO) Annual Meeting in Chicago, Illinois, on June 3-7, 2016, and as a poster at the American Society of Hematology (ASH) Annual Meeting in San Diego, California, on December $3-6,2016$

Study concept and design were contributed by Latremouille-Viau and Guerin, along with the other authors. Gagnon-Sanschagrin and Dea took the lead in data collection, assisted by the other authors, and data interpretation was performed by Cohen and Joseph, along with the other authors. The manuscript was written by Latremouille-Viau, along with the other authors, and revised by Joseph, along with the other authors.

\section{ACKNOWLEDGMENTS}

Manuscript drafts were prepared by a professional medical writer, Shelley Batts, PhD, an employee of Analysis Group. The authors thank Johannes Wolff, $\mathrm{MD}$, an employee of Novartis, for review of the data and scientific input.

\section{REFERENCES}

1. National Cancer Institute, Surveillance, Epidemiology, and End Results Program. SEER stat fact sheets: chronic myeloid leukemia (CML). 2016. Available at: http://seer.cancer.gov/statfacts/html/cmyl.html. Accessed December 31, 2016.

2. Howlader N, Noone A, Krapcho M, et al., eds. SEER cancer statistics review, 1975-2013. National Cancer Institute. Bethesda, MD. Updated September 12, 2016. Available at: http://seer.cancer.gov/csr/1975_2013/. Accessed December 31, 2016.

3. Kurzrock R, Kantarjian HM, Druker BJ, Talpaz M. Philadelphia chromosome-positive leukemias: from basic mechanisms to molecular therapeutics. Ann Intern Med. 2003;138(10):819-30.

4. Goldman JM, Melo JV. Chronic myeloid leukemia-advances in biology and new approaches to treatment. N Engl J Med. 2003;349(15):1451-64.

5. Havelange V, Demoulin JB. Review of current classification, molecular alterations, and tyrosine kinase inhibitor therapies in myeloproliferative disorders with hypereosinophilia. J Blood Med. 2013;4:111-21.

6. GLEEVEC (imatinib mesylate) tablets for oral use. Novartis. 2010. Available at: http://www.accessdata.fda.gov/drugsatfda_docs/ label/2008/021588s024lbl.pdf. Accessed December 31, 2016.

7. SPRYCEL (dasatinib) tablet for oral use. Bristol-Myers Squibb Company. 2010. Available at: http://www.accessdata.fda.gov/drugsatfda_docs/ label/2010/021986s7s8lbl.pdf. Accessed December 31, 2016.

8. Tasigna (nilotinib) capsules. Novartis. 2010. Available at: http://www. accessdata.fda.gov/drugsatfda_docs/label/2010/022068s004s005lbl.pdf. Accessed December 31, 2016.

9. Kantarjian H, O'Brien S, Jabbour E, et al. Improved survival in chronic myeloid leukemia since the introduction of imatinib therapy: a single-institution historical experience. Blood. 2012;119(9):1981-87.

10. Erba HP. Molecular monitoring to improve outcomes in patients with chronic myeloid leukemia in chronic phase: importance of achieving treatment-free remission. Am J Hematol. 2015;90(3):242-49.

11. Etienne G, Dulucq S, Nicolini FE, et al. Achieving deeper molecular response is associated with a better clinical outcome in chronic myeloid leukemia patients on imatinib front-line therapy. Haematologica.

2014;99(3):458-64

12. Goldberg SL, Chen L, Guerin A, et al. Association between molecular monitoring and long-term outcomes in chronic myelogenous leukemia patients treated with first line imatinib. Curr Med Res Opin. 2013;29(9):1075-82.

13. Bauer S, Romvari E. Interpreting molecular monitoring results and international standardization in chronic myeloid leukemia. J Adv Pract Oncol. 2012:3(3):151-60
14. Cross NC. Standardisation of molecular monitoring for chronic myeloid leukaemia. Best Pract Res Clin Haematol. 2009;22(3):355-65.

15. Hughes T, Deininger M, Hochhaus A, et al. Monitoring CML patients responding to treatment with tyrosine kinase inhibitors: review and recommendations for harmonizing current methodology for detecting BCR-ABL transcripts and kinase domain mutations and for expressing results. Blood. 2006;108(1):28-37

16. Baccarani M, Deininger MW, Rosti G, et al. European LeukemiaNet recommendations for the management of chronic myeloid leukemia: 2013. Blood. 2013;122(6):872-84

17. Radich JP, Deininger M, Abboud CN, et al. NCCN Clinical Practice Guidelines in Oncology: chronic myelogenous leukemia, version 1.2016. Available at: https://www.nccn.org/professionals/physician_gls/pdf/aml.pdf. Accessed March 15, 2016

18. Guérin A, Chen L, Dea K, Wu EQ, Goldberg SL. Association between regular molecular monitoring and tyrosine kinase inhibitor therapy adherence in chronic myelogenous leukemia in the chronic phase. Curr Med Res Opin. 2014;30(7):1345-52.

19. Guérin A, Chen L, Dea K, Wu EQ, Goldberg SL. Economic benefits of adequate molecular monitoring in patients with chronic myelogenous leukemia. J Med Econ. 2014;17(2):89-98

20. Chen L, Guérin A, Xie J, et al. Monitoring and switching patterns of patients with chronic myeloid leukemia treated with imatinib in community settings: a chart review analysis. Curr Med Res Opin. 2012;28(11):1831-39.

21. Wu EQ, Johnson S, Beaulieu N, et al. Healthcare resource utilization and costs associated with non-adherence to imatinib treatment in chronic myeloid leukemia patients. Curr Med Res Opin. 2010;26(1):61-69.

22. The American Medical Association. CPT 2013 Professional edition package [software]. 2013.

23. Peterson AM, Nau DP, Cramer JA, Benner J, Gwadry-Sridhar F, Nichol M. A checklist for medication compliance and persistence studies using retrospective databases. Value Health. 2007;10(1):3-12.

24. U.S. Bureau of Labor Statistics. Consumer Price Index (CPI) medical care component. 2015. Available at: http://www.bls.gov/cpi/. Accessed December 31, 2016

25. Bagdonavicius VN, Nikulin, N. On goodness-of-fit for homogeneity and proportional hazard. Appl Stoch Model Bus. 2006;22(5-6):607-19.

26. Hosmer DWL, Lemesbow S. Goodness of fit tests for the multiple logistic regression model. Commun Stat Theory Methods. 1980;9(10):1043-69.

27. White H. A heteroskedasticity-consistent covariance matrix estimator and a direct test for heteroskedasticity. Econometrica. 1980;48(4):817-38.

28. Stock JH, Watson MW. Introduction to Econometrics. 2nd ed. Boston, MA: Pearson/Addison Wesley; 2007.

29. Darkow T, Kadlubek PJ, Shah H, Phillips AL, Marton JP. A retrospective analysis of disability and its related costs among employees with chronic obstructive pulmonary disease. J Occup Environ Med. 2007;49(1):22-30.

30. Charlson ME, Pompei P, Ales KL, MacKenzie CR. A new method of classifying prognostic comorbidity in longitudinal studies: development and validation. J Chronic Dis. 1987;40(5):373-83.

31. Andrews DW, Buchinsky M. A three-step method for choosing the number of bootstrap repetitions. Econometrica. 2000;68(1):23-51.

32. Davidson R, MacKinnon JG. Econometric Theory and Methods. Vol 5. New York: Oxford University; 2004

33. Partridge AH, Avorn J, Wang PS, Winer EP. Adherence to therapy with oral antineoplastic agents. J Natl Cancer Inst. 2002;94(9):652-61.

34. Karve S, Cleves MA, Helm M, Hudson TJ, West DS, Martin BC. Good and poor adherence: optimal cut-point for adherence measures using administrative claims data. Curr Med Res Opin. 2009;25(9):2303-10. 
35. Cabana MD, Rand CS, Powe NR, et al. Why don't physicians follow clinical practice guidelines? A framework for improvement. JAMA 1999;282(15):1458-65.

36. Kolibaba KS. Molecular monitoring of response in patients with chronic myeloid leukemia. Manag Care. 2013;22(7):40, 50-61.

37. Goldberg SL, Akard LP, Dugan MJ, Faderl S, Pecora AL. Barriers to physician adherence to evidence-based monitoring guidelines in chronic myelogenous leukemia. J Oncol Pract. 2015;11(3):e398-404.

38. Centers for Medicare and Medicaid. Clinical laboratory fee schedule. 2016. Available at: https://www.cms.gov/Medicare/Medicare-Fee-for-ServicePayment/ClinicalLabFeeSched/Clinical-Laboratory-Fee-Schedule-Files.html. Access January 11, 2017.

39. Baccarani M, Soverini S. Molecular response in CML: where is the bar? Blood. 2014;124(4):469-71.

40. Branford S, Fletcher L, Cross NC, et al. Desirable performance characteristics for BCR-ABL measurement on an international reporting scale to allow consistent interpretation of individual patient response and comparison of response rates between clinical trials. Blood. 2008;112(8):3330-38.

41. Hughes TP, Hochhaus A, Branford S, et al. Long-term prognostic significance of early molecular response to imatinib in newly diagnosed chronic myeloid leukemia: an analysis from the International Randomized Study of Interferon and STI571 (IRIS). Blood. 2010;116(19):3758-65.
42. Neelakantan P, Gerrard G, Lucas C, et al. Combining BCR-ABLl transcript levels at 3 and 6 months in chronic myeloid leukemia: implications for early intervention strategies. Blood. 2013;121(14):2739-42.

43. Brümmendorf TH, Kantarjian HM, Gambacorti-Passerini C, et al. Assessment of early molecular response as a predictor of long-term clinical outcomes in the phase 3 BELA study. Blood. 2012;120(21):Abstract 69.

44. Kantarjian H, Schiffer C, Jones D, Cortes J. Monitoring the response and course of chronic myeloid leukemia in the modern era of BCR-ABL tyrosine kinase inhibitors: practical advice on the use and interpretation of monitoring methods. Blood. 2008;111(4):1774-80.

45. Radich JP. Molecular monitoring of patients with chronic myeloid leukemia: clinical examples from a non-trial setting. Clin Lymphoma Myeloma. 2009;9(Suppl 4):S391-94.

46. Sokal JE, Cox EB, Baccarani M, et al. Prognostic discrimination in "good-risk" chronic granulocytic leukemia. Blood. 1984;63(4):789-99.

47. Oken MM, Creech RH, Tormey DC, et al. Toxicity and response criteria of the Eastern Cooperative Oncology Group. Am J Clin Oncol. 1982;5(6):649-55. 
Health Care Resource Utilization and Costs in Patients with Chronic Myeloid Leukemia with Better Adherence to Tyrosine Kinase Inhibitors and Increased Molecular Monitoring Frequency

APPENDIX A Codes Used to Search for Procedures and Diagnoses

\begin{tabular}{|c|c|c|}
\hline CPT Code & ICD-9-CM Code & Description \\
\hline- & $205.1 x$ & Chronic myeloid leukemia (CML) \\
\hline- & 205.12 & CML relapse \\
\hline- & 205.11 & CML remission \\
\hline- & $\begin{array}{l}\text { 196.xx, 197.xx, 198.xx, 199.xx, 209.7x, } 789.51 \\
\text { 140.xx to 165.xx } \\
\text { 179.xx to 195.xx } \\
\text { 170.xx, 171.xx, 172.xx, 174.xx, 175.xx, 176.xx, 209.0x, 209.1x, 209.2x, } \\
\text { 209.3x, 258.01, 258.02, 258.03 }\end{array}$ & Metastatic cancer or solid tumor \\
\hline- & V70.7 & Participation in a clinical trial \\
\hline $38240,38241,38242$ & $\begin{array}{l}\text { Procedure: } 41.00,41.01,41.02,41.03,41.04,41.05,41.07,41.08,41.09 \\
\text { Diagnosis: V42.81, V42.82, 238.77, 279.5x, 996.8x }\end{array}$ & Hematopoietic stem cell transplant \\
\hline
\end{tabular}


Health Care Resource Utilization and Costs in Patients with Chronic Myeloid Leukemia with

Better Adherence to Tyrosine Kinase Inhibitors and Increased Molecular Monitoring Frequency

APPENDIX B Molecular Monitoring and CPT Codes Used Before and After 2013

1. Definition of Molecular Monitoring and CPT Codes Used Before and After 2013

\begin{tabular}{|c|c|}
\hline CPT Code & Description \\
\hline \multicolumn{2}{|c|}{ Before $2013^{a}$} \\
\hline 83891 & Isolation or extraction of highly purified nucleic acid, each nucleic acid type (i.e., DNA or RNA) \\
\hline 83892 & Enzymatic digestion, each enzyme treatment \\
\hline 83896 & Nucleic acid probe, each 20 \\
\hline 83898 & Amplification, target, each nucleic acid sequence \\
\hline 83900 & Amplification, target, multiplex, first 2 nucleic acid sequences \\
\hline 83901 & Amplification, target, multiplex, each additional nucleic acid sequence beyond 2 \\
\hline 83902 & Reverse transcription \\
\hline \multicolumn{2}{|l|}{ After 2013} \\
\hline 81206 & BCR/ABL1 (t (9;22)) (e.g., chronic myelogenous leukemia) translocation analysis; major breakpoint, qualitative or quantitative \\
\hline 81207 & BCR/ABL1 (t (9;22)) (e.g., chronic myelogenous leukemia) translocation analysis; minor breakpoint, qualitative or quantitative \\
\hline 81208 & BCR/ABL1 (t (9;22)) (e.g., chronic myelogenous leukemia) translocation analysis; other breakpoint, qualitative or quantitative \\
\hline
\end{tabular}

\section{Scheme and CPT Codes Used for Identifying Molecular Monitoring Before 2013}
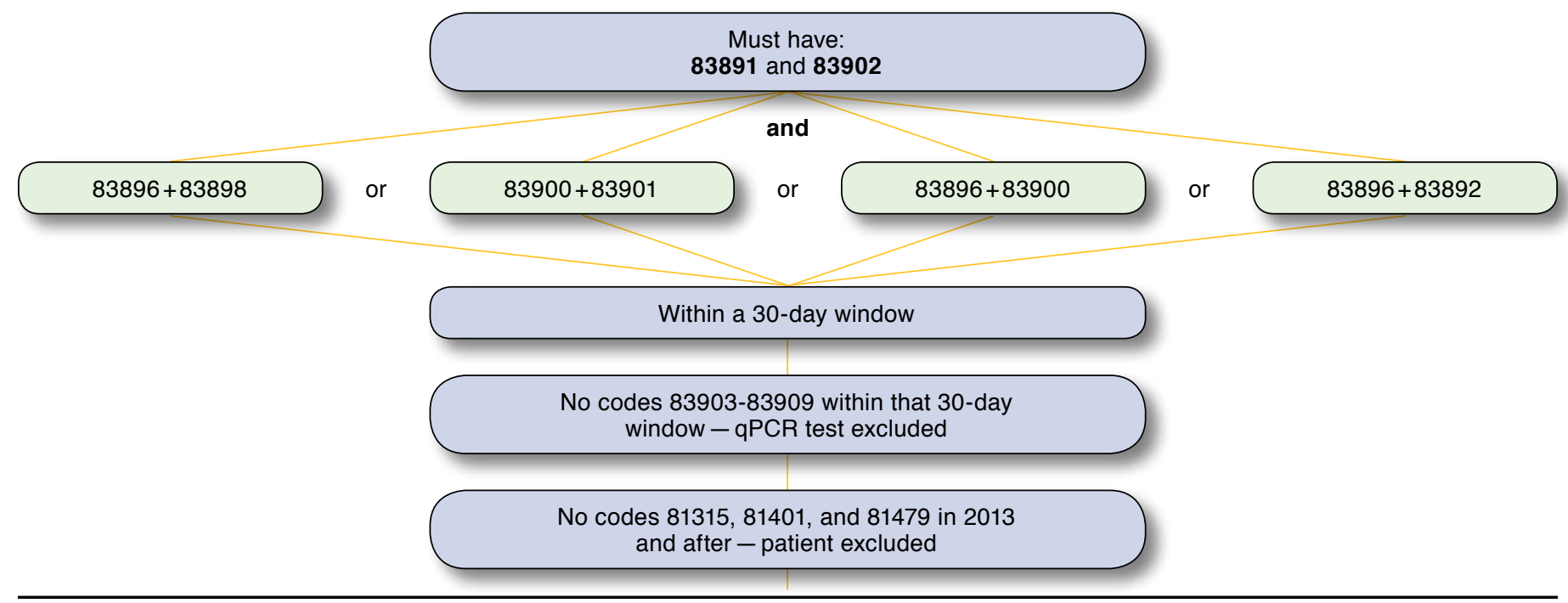

Note: Code descriptions listed in Appendix B1.

$C P T=$ Current Procedural Terminology; $q P C R=$ quantitative real-time polymerase chain reaction

\section{APPENDIX B REFERENCES}

- Quest Diagnostics. BCR/ABLl Gene rearrangement, quantitative, PCR. 2015. Available at: http://www.questdiagnostics.com/testcenter/TestDetail. action?ntc=91065. Accessed December 31, 2016

- CML HealthCare. Test specification guide. October 31, 2016. Available at: http://cmlhealthcare.com/wp-content/uploads/2016/10/TEST-

SPECIFICATION-GUIDE_31OCT2016.pdf. Accessed December 31, 2016.

- Mayo Clinic. CPT codes for molecular pathology. 2013.

- Stanford University Medical Center. Molecular 2013 CPT code. 2013
- AmeriPath. BCR/ABL quantitative PCR. 2013

- Mayo Clinic. BCR/ABL1, qualitative, diagnostic assay. 2015. 2015. Available at: http://www.mayomedicallaboratories.com/test-catalog/Clinical+ and+Interpretive/89006. Accessed December 31, 2016

- Diagnostic Laboratory of Oklahoma Clinic. 2012 AMA changes in CPT coding. 2012.

- Cleveland Clinic Laboratories. 2013 new molecular CPT codes. 2013. Available at: http://portals.clevelandclinic.org/portals/66/pdf/2013_New_ Molecular_CPT_Codes.pdf. Accessed December 31, 2016. 